\title{
Sylvie Vartan - 50 ans de bonheur? \\ Stationen in der Karriere eines yé-yé-Idols
}

\author{
Andreas BONNERMEIER (Mainz)
}

\section{Summary}

About 50 years ago, Sylvie Vartan was one of the major stars of the French chanson yé-yé. Being the perfect example of promulgation through the mass media, she quickly received the status of an idol and became one of the favorite copains of French adolescents in the 1960s. As one of the few real stars of the yé-yé movement, she managed to continue her career up to the present day: first by adopting the American show model, then by engaging in projects with new authors and representatives of the new French chanson. Success was not always part of the show, but Vartan found a way to conserve parts of her public even in the new millennium. Is this the reason why she can be considered an idol unaffected by time?

Das chanson yé-yé ist eine der Stilrichtungen, die das französische Chanson der 1960er Jahre mitgeprägt haben und dem viele InterpretInnen den Start in eine musikalische Karriere verdanken. Doch nur wenige von ihnen haben es geschafft, sich auf Dauer einen Platz in der französischen Chansonlandschaft zu sichern. Neben Johnny Hallyday und Françoise Hardy gehört Sylvie Vartan zu jenen Stars des chanson yé-yé, die noch heute regelmäßig neue Produktionen veröffentlichen und Konzerte geben. Doch sind ihr Stellenwert und ihre Popularität noch genauso hoch wie vor 50 Jahren? Ist es der Interpretin gelungen, ihre Karriere vom Idol der 1960er Jahre in eine dauerhafte Präsenz im französischen Chanson umzuwandeln oder bleibt sie auf das chanson yé-yé reduziert? Der nachfolgende Aufsatz zeichnet die unterschiedlichen Phasen der Karriere der Interpretin von den Anfängen im chanson yé-yé bis heute nach. Zum besseren Verständnis soll dabei in einem gesonderten Kapitel auch auf Faktoren wie die Mediatisierung und Idolisierung der SängerInnen eingegangen werden, die gerade in jener Zeit eine entscheidende Rolle spielen. 


\section{Das chanson yé-yé und der Zeitgeist}

Ehe wir uns dem konkreten Beispiel Sylvie Vartans zuwenden, ist es zunächst erforderlich, die Rolle und den Kontext des chanson yé-yé in den 1960er Jahren zu analysieren, um dem Zeitgeist jener Epoche auf die Spur zu kommen. In der Tat ist die erste Hälfte der 1960er Jahre in Frankreich von einem besonderen sozialen Klima geprägt: Einerseits läutet diese Epoche das Ende der rigiden Nachkriegsgesellschaft mit ihren konservativen Moral- und Wertvorstellungen ein, die durch die Jahre des wirtschaftlichen Aufschwungs nachhaltig verändert wurde. Andererseits dauert es noch bis zu den Protestbewegungen im Mai 1968, bis die junge Generation der Gesellschaft in Frankreich nachhaltige Änderungen abringt. $\mathrm{Zu}$ diesem Zeitpunkt jedoch hat das chanson yé-yé seine Hochzeit bereits längst überschritten und muss neuen Musikrichtungen weichen. Dennoch hinterlassen die Jahre der Trente Glorieuses $^{2}$ auch im französischen Chanson deutliche Spuren. Zum einen führen der wirtschaftliche Aufschwung und der damit verbundene Anstieg des Lebensstandards zu einem vermehrten Konsum, der sich auch im Bereich der Unterhaltung niederschlägt: So erreicht der Verkauf von Schallplatten, vor allem der damals sehr beliebten Singles, bis dato nie dagewesene Rekordstände, was freilich auch durch technische Neuerungen wie tragbare Plattenspieler und Transistorradios, die für relativ wenig Geld zu erwerben sind, und die Einführung der Jukebox nach amerikanischem Vorbild massiv forciert wird. Zum anderen ist es das erste Mal in der französischen Geschichte, dass sich die Jugendlichen deutlich von der Generation ihrer Eltern absetzen und sich eine eigene Welt schaffen: „Ils [les adolescents] imposent leur façon de s'habiller, de se coiffer, la musique qu'ils aiment et sur laquelle ils dansent [...]. “ (Lesouëf 2013, 65) Die Tatsache, dass zum ersten Mal Jugendliche auch Taschengeld beziehen, eröffnet ihnen die Möglichkeit, an dem neu entstehenden Musikmarkt zu partizipieren und sich damit auch vom Musikgeschmack ihrer Eltern abzugrenzen:
Pour la première fois sans doute dans l'histoire de notre pays, la jeunesse, accède à la consommation avant d'être passée par le stade de la production. [...] La musique - et par là même la chanson - devient le véhicule identitaire privilégié de cette aspiration à l'hédonisme, favorisée par l'expansion économique. (Dillaz 2005, 92)

Die Musikindustrie wiederum versteht sich im Schulterschluss mit den Medien bestens darauf, diese neue Käuferschicht zu erobern: So werden neben den nun auch für die Jugendlichen erschwinglichen Singles spezielle Radiosendungen kreiert, die sich explizit an dieses Publikum richten. Das 1959 ins Leben gerufene Salut les copains ist die sicherlich bekannteste Sendung aus jenen Jahren und hat in Spitzenzeiten mehr als 4 Millionen Zuhörer (Oger 2013, 120), die Abkürzung SLC ist in der jungen Generation in aller Munde. Der Verleger Daniel Filipacci erkennt in diesem Zusammenhang sehr schnell das wirtschaftliche Potential und bringt 1962 die gleichnamige Zeitschrift Salut les copains heraus, die ein durchschlagender Erfolg wird und nicht zuletzt dazu führt, dass der Begriff ,copain' in der ersten Hälfte der 1960er Jahre eine ganze Generation prägt: „La musique est sans conteste 
le déclencheur de cette culture ,jeune' qui va insuffler, tous domaines confondus, aux années soixante une formidable effervescence." (Oger 2013, 122)

Interessant ist in diesem Zusammenhang, dass die Texte des chanson yé-yé vielfach äußerst konventionell sind und keineswegs die Jugendlichen dazu animieren, den offenen Bruch mit der Generation ihrer Eltern zu suchen, sondern eher Verhaltensmodelle für eine Zukunft projizieren, die letzten Endes denen der älteren Generationen ähneln:

Ce qui est frappant, en effet, c'est que le discours véhiculé par les chansons des yé-yé n'est pas spécialement un discours de rupture face à la génération précédente (contrairement à la musique [...]), qu'il est même parfois très conformiste et que, d'une certaine façon, il prépare les fils et les filles à se comporter plus tard comme leurs parents. (Calvet 2013, 144-145)

Auf der textlichen Ebene zeigt sich mehr als deutlich, dass das Frankreich in der Zeit vor Mai 68 noch konservative Züge trägt, was aber weniger überrascht, wenn man bedenkt, dass viele AutorInnen des chanson yé-yé auch schon in den Jahren zuvor als Chansontexter arbeiten (wie z.B. Pierre Delanoë) oder gar als A-C-I tätig sind wie etwa Charles Aznavour oder Serge Gainsbourg. ${ }^{3}$ Das Chanson „La plus belle pour aller danser“ von Sylvie Vartan steht beispielhaft für diesen Sachverhalt, wie die konventionellen Formulierungen am Beginn des Chansons zeigen: „Ce soir, je serai la plus belle / Pour aller danser / danser / Pour mieux évincer toutes celles / Que tu as aimées / Aimées / Ce soir je serai la plus tendre / Quand tu me diras / Diras / Tous les mots que je veux entendre [...]" (Saka 2001, 640).

Was nun die InterpretInnen des chanson yé-yéangeht, kommt noch ein weiteres zentrales Element hinzu: In der Regel sind diese im selben Alter wie die Zielgruppe, d.h. sie haben selbst die Schule gerade beendet oder sind noch Schüler, haben dieselben Interessen wie ihre Fans und bieten diesen dadurch Projektionsflächen hinsichtlich Kleidung, Frisuren und Aussehen. Sie werden aber auch als sympathische Stars von nebenan, ja sogar als ,copains ' betrachtet: „Entre ces artistes qui ont entre 16 et 18 ans, l'âge de leurs fans, l'empathie est totale: pour les jeunes qui les appellent par leurs prénoms, les tutoient, les chanteurs et chanteuses ne sont plus des stars inaccessibles; ce sont des copains qui les comprennent." (Oger 2013, 120) Insofern entsprechen diese InterpretInnen dem Zeitgeist der Jugendlichen, ja sie verkörpern ihn sogar und werden damit zu Vorbildern oder gar zu Idolen, die es nachzuahmen gilt.

\section{Medienpräsenz und Idolisierung}

Wie bereits deutlich wurde, spielen die Medien für das chanson yé-yé und seine Stars eine erhebliche Rolle, was nicht zuletzt daran liegt, dass die Idolisierung bestimmter Sängerinnen und Sänger auch durch die Medien massiv gefördert wird: So prägen die Massenmedien wie Fernsehen, Radio und Zeitungen deren öffentliches Bild, das sich sehr schnell zu einem bestimmten Image verfestigt; sie bieten Identifikationsmodelle an und tragen mitunter zur 
Herausbildung von modernen Mythen rund um eine Sängerin oder einen Sänger bei. ${ }^{4}$ Auf diese Weise werden die Medien zu einem wichtigen Bindeglied zwischen Chanson/Interpret auf der einen und dem Publikum auf der anderen Seite: „Le chanteur et le public entretiennent en effet une relation très marquée par l'influence des médias." (Hölz 1988, 347) Sei es durch Interviews und Reportagen in der Presse, sei es durch das Vorstellen einer neuen Produktion, die Medienpräsenz ist für einen Sänger unumgänglich, um seine Musik dem Publikum zu offerieren. Doch diese Promotion wird noch durch eine weitere wichtige Ebene ergänzt: Sowohl Signierstunden in Geschäften oder nach Konzerten als auch sogenannte „Homestories' in Presse und Fernsehen, bei denen die Stars einen Blick in ihr vermeintlich authentisches Privatleben zulassen, gehören zum Business und vermitteln den Eindruck, man habe es mit einem Star ,zum Anfassen' zu tun. Einmal mehr werden die InterpretInnen dadurch zu Identifikationsmodellen, was mitunter dazu führt, dass die Chansons selbst in den Hintergrund treten: „Ce n'est pas la chanson mais la star qui importe au public. Le degré de popularité devient le critère d'appréciation et de qualité." (Hölz 1988, 357) Doch gilt es gerade auch im Bereich der Medienpräsenz zwischen der realen Person und der performance person $a^{5}$ zu unterscheiden: So ist der Star mit seinem u.a. von den Medien geprägten Image eindeutig dem Bereich der performance persona zuzuordnen, die zwar Gemeinsamkeiten mit der realen Person haben kann, aber nicht haben muss. Die Unterscheidung zwischen den beiden Personen ist dabei mitunter gerade auch für Medien und Fans schwierig. Es darf jedoch davon ausgegangen werden, dass in den Medien in erster Linie die performance persona im Vordergrund steht, welche wiederum von den Medien selbst mit menschlichen Zügen, die der vermeintlich realen Person zuzuordnen sind, ausgestattet wird; die bereits erwähnten Blicke ins Privatleben oder Berichte über private Schicksalsschläge oder freudige Ereignisse sind Beispiele, die sich in den 1960er Jahren sehr häufig in den französischen Medien finden.

Dass diese Mediatisierung in Frankreich mit dem chanson yé-yé einen Höhepunkt erlebt, ist kein Zufall: Auch wenn die Verehrung von SängerInnen und SchauspielerInnen unter dem Etikett ,Diva' weit in die Vergangenheit zurückreicht (man denke an die Schauspielerin Sarah Bernardt oder die OpernsängerInnen Enrico Caruso und Maria Callas) und wenige Jahre vor dem chanson yé-yé die Stilrichtung des Rock'n'Roll Idole wie Elvis Presley hervorbringt, so trägt doch gerade die speziell im Kontext des chanson yé-yé entstandene Musikpresse wie Salut les Copains entscheidend dazu bei, die damals noch jungen InterpretInnen zu Stars zu machen, indem sie ihnen eine permanente Plattform bei ihrem Publikum bietet. Dabei werden diese verstärkt auch außerhalb der Bühne zu Objekten des öffentlichen Interesses; die Begegnung zwischen KünstlerInnen und Publikum ist folglich nicht mehr nur auf die Bühne beschränkt:

De façon symptomatique, le yéyé, qui pour [Edgar] Morin est la première illustration parfaite de la 'vedettisation de l'interprète', va de pair, d'une part avec l'expansion de la presse musicale, qui présente l'interprète comme une personne que l'on peut saisir visuellement et, ce faisant, oppose à la scène, le lieu jusqu'à présent unique où interprète et public se rencontrent, une forme de transmission visuelle [...]. (Bügler 1988, 369) 
So werden die VertreterInnen des chanson yé-yé von ihren Fans in einem nie dagewesenen Ausmaß und in einer Weise verehrt, die einem religiösen Kult nahekommt. Dadurch erfährt der Begriff ,Idol', der im ursprünglichen Sinn eine „représentation d'une divinité que l'on adore et qui est l'objet d'un culte au même titre que la divinité elle-même "“6 bezeichnet, eine semantische Verschiebung, durch die die Sängerinnen und Sänger auf eine Stufe mit göttlichen Wesen gestellt werden. Der Trésor de la Langue Française definiert diese neue Art von Idol folglich als „personne ou chose intensément admirée et faisant l'objet d'une sorte de vénération“ und fügt präzisierend hinzu: „Vedette du spectacle, de la chanson ou personnalité à la mode dont l'apparition suscite une frénésie dans le public." ${ }^{\text {"7 }}$ So werden in Frankreich erstmals die Stars des chanson yé-yé mit dem neuen Etikett ,Idol" versehen: „Tous les jours au sortir de la classe, les adolescents du pays collent l'oreille à un transistor pour tout savoir sur ceux que l'on désigne désormais comme idoles." (Lesouëf 2013, 69) Zugleich jedoch trägt die Berichterstattung in den Medien auch dazu bei, dass das Publikum den Eindruck bekommt, seine Stars und Idole würden in einem eigenen Universum leben und gleichsam privilegiert sein:

Les stars partagent les mêmes conditions d'existence. Évoluant uniquement entre elles, elles délimitent un espace social clos et privilégié qui les place alors dans une sorte de cité olympienne: les vedettes naviguent dans un royaume aquarium, éclairé artificiellement, étouffant, confiné où seuls les êtres de cette espèce survivent [...]. (Brunel 1999, 747)

Dies führt schließlich dazu, dass die Stars des chanson yé-yéin der kollektiven Wahrnehmung einen Platz einnehmen, der bislang dem Adel und anderen Persönlichkeiten der sogenannten ,besseren Gesellschaft vorbehalten war. Es handelt sich mit anderen Worten um „un phénomène de société dans lequel les amours de Sylvie et Johnny prennent la place jusque-là réservée à Margaret ou à Soraya [...]" (Calvet 2013, 140). Die bereits erwähnte Trennung zwischen realer Person und performance persona tritt dabei in den Hintergrund und wird bei der kollektiven Verehrung nur noch bedingt wahrgenommen.

Am Beispiel Sylvie Vartans lässt sich dieses Phänomen denn auch besonders gut zeigen: Nachdem die aus Bulgarien stammende Interpretin (sie wurde 1944 in Sofia geboren und übersiedelte 1952 mit ihren Eltern und ihrem Bruder nach Paris) 1961 als Duettpartnerin von Frankie Jordan mit „Panne d'essence“ einen ersten Hit gelandet hat, bricht sie die Schule ab und nimmt ihre erste ,eigene 'Single, „Quand le film est triste“, auf. Mit Produktionen wie „Est-ce que tu le sais“, „Si je chante“, „La locomotion“ und „Comme un garçon“ wird sie über Nacht zu einem der Idole des chanson yé-yé. Zu einem ihrer größten Hits überhaupt wird 1964 „La plus belle pour aller danser“, das aus der Feder von Charles Aznavour und Georges Garvarentz stammt und für den Film Cherchez l'idole geschrieben wurde. Es folgen zahlreiche weitere Chansons, deren Texte häufig Übersetzungen von amerikanischen Songs der Zeit sind und bei denen die Musik eine wichtigere Rolle als der Text spielt. Doch Sylvie Vartan wird auch rasch zu einem Liebling der Medien: Sei es die Beziehung und Ehe mit ihrem Sangeskollegen Johnny Hallyday, die Geburt des gemeinsamen Sohnes David 
1966, seien es zwei schwere Autounfälle in den Jahren 1968 und 1970, die u.a. chirurgische Eingriffe zur Wiederherstellung des Gesichtes zur Folge haben, sei es die Scheidung von Hallyday 1980: Die Medien sind stets dabei und berichten ausführlich über die Interpretin. Auf diese Weise vermitteln sie den Eindruck, ein realistisches Bild der realen Person Sylvie Vartan zu zeichnen, das jedoch in Wirklichkeit der performance persona zuzuordnen ist. Den Höhepunkt des Medienhypes um Hallyday und Vartan stellt dabei sicherlich die Hochzeit der beiden im Jahre 1965 dar, die alleine der Zeitschrift Salut les copains eine Auflage von 1,5 Millionen Exemplaren beschert (Lesouëf 2013, 70) und Hallyday und Vartan zu einem „phénomène de société" (Jelot-Blanc 2014, 155) macht. Die gigantische Vermarktungsmaschinerie nutzt die Popularität der Interpretin indes auf vielfältige Weise, beispielsweise in der Werbung für einen Friseursalon in Paris, vor allem aber auch im Bereich der Mode, indem sie u.a. Vartans Namen auf Kleider, Taschen und Brillen druckt (Dillaz 2005, 123), die Interpretin als Mannequin für berühmte Couturiers wie Dior ${ }^{8}$ auftreten lässt und ihre zahlreichen Fans damit zum Konsum anregt. Die Interpretin wird damit zur Modeikone und tritt in die Fußstapfen Brigitte Bardots: Nicht umsonst imitieren tausende junger Französinnen in den 1960er Jahren den Look Sylvie Vartans, nämlich „une coupe au carré un peu ,gonflant', un trait d'eyeliner" (Ogier 2013, 151), aber auch ihren stets wechselnden Kleidungsstil. Vartan eröffnet 1965 schließlich gar eine eigene Boutique und bringt eine eigene Modekollektion heraus. Es mag daher kaum verwundern, dass Vartan auch Protagonistin einer eigenen Radioserie bei RTL mit dem Titel Les malheurs de Sylvie wird, aber auch in den damals sehr beliebten Fotoromanen in Zeitschriften wie Nous deux, die vor allem beim weiblichen Publikum hoch im Kurs stehen, einen Platz erhält. ${ }^{9}$

Zum Sinnbild der yé-yé-Periode wird schließlich Vartans Titel „2'35 de bonheur“ aus dem Jahre 1967, der jedoch einen Wandel im Repertoire der Interpretin einleitet: Mit „La Maritza" aus dem Jahre 1968, das eine autobiographische Dimension beinhaltet und einen anderen musikalischen Stil aufweist, beginnt der Weg der Interpretin in die Zeit nach dem chanson yé-yé.

\section{Phase I: Bühnenshows à l'américaine}

Nach dem Ende des chanson yé-yé stellt sich für Sylvie Vartan wie für ihre zahlreichen KollegInnen die Frage nach einer Neuausrichtung ihrer Karriere, wobei sie im Gegensatz zu vielen anderen den Vorteil hat, den Status eines One-Hit-Wonders längst hinter sich gelassen zu haben. So zeigt sie schon 1968 eine völlig neue Facette im Pariser Olympia: In Anlehnung an die großen Tanzrevuen der 1920er und 1930er Jahre präsentiert sie Balladen und Chansons in neuen Arrangements, die sich deutlich vom chanson yé-yéabheben. Die Inszenierung der Bühnenshow setzt dabei einen eigenen Akzent: „Pour marquer cette émancipation, le spectacle se termine par une descente d'escalier, celui des revues de l'entre-deux-guerres." (Calvet 2006, 476) Doch dieses Programm ist nur die Vorstufe zu einer Weiterentwicklung in den 1970er Jahren, in denen Vartan mit Bühnenshows im amerikanischen Stil für 
Furore sorgt. Als eine der ersten Sängerinnen in Frankreich etabliert sie diesen neuen Typ von Bühnenshow, in dem neben dem Gesang auch Tanz- und Showeinlagen eine tragende Rolle spielen: „choréographies travaillées, ballets colorés, éclairages savants et chansons aux mélodies évidentes“(Saka/Plougastel 1999, 425) bilden ein Erfolgsrezept, das in den Jahren 1975, 1977 und 1978 für ausverkaufte Vorstellungen im Pariser Palais des Congrès sorgt. Vartan ist mit diesem Kurswechsel allerdings nicht die einzige Interpretin des chanson yé-yé, denn auch Kollegen wie Claude François oder Sheila versuchen sich in dieser Sparte: „Côté français, diverses vedettes en perte de vitesse [...] en profitent pour relancer leur carrière." (Dillaz 2005, 347) Auch andere Interpretinnen wie Dalida (die 1980 im Palais des Sports eine ähnliche Show präsentiert) werden zur Konkurrenz für Vartan, während sich Johnny Hallyday auf ein rockiges Repertoire verlegt und Françoise Hardy einen ganz eigenen Weg einschlägt.

Es ist jedoch unübersehbar, dass die Popularität Sylvie Vartans in den Medien deutlich nachlässt, woran auch ein 1973 mit ihrem Ehemann Johnny Hallyday aufgenommenes Duett "J'ai un problème“ nichts ändert: Das Chanson wird zwar zu einem Hit, wird von Vartan selbst im Nachhinein vor dem Hintergrund ihrer damals bereits problematischen Ehe mit Hallyday aber als „ridicule“ (Vartan 2004, 260) bezeichnet. Das Chanson schließt somit einen Bogen zur Medienpräsenz der beiden Sänger in den 1960er Jahren, kann es doch als eine Art Fortsetzung der vermeintlich authentischen Berichterstattung aus deren Leben interpretiert werden. Dadurch kann es gleichzeitig als Beispiel dienen, wie die bereits erwähnten Dimensionen zwischen realer Person, performance person und character im Sinne Philip Auslanders verschwimmen.

Was die künstlerische Produktion Sylvie Vartans aus den 1970er und frühen 1980er Jahren angeht, ist es wenig verwunderlich, dass es sich einmal mehr vielfach um französische Adaptionen von Songs aus dem anglophonen Raum handelt, wie z.B. „Toi le garçon“, „Bye bye Leroy Brown“, „Qu'est-ce qui fait pleurer les blondes“, „Le temps du swing“ oder „Petit rainbow". Auch bei diesen Produktionen liegt der Focus weniger auf dem Text als auf der Melodie, die sich für die szenische und tänzerische Umsetzung auf der Showbühne eignen muss. Viele dieser Chansons haben ihren Ursprung auch im damals aktuellen Musikstil des Disco, der in der zweiten Hälfte der 1970er Jahre auch in Frankreich für Furore sorgt. Nur einzelne Chansons im Repertoire Vartans, wie beispielsweise „Nicolas“ von 1979, das eine Brücke zu den osteuropäischen Wurzeln der Interpretin schlägt, bilden dazu einen Kontrast. Es lässt sich nur schwer übersehen, dass die Karriere der Interpretin stagniert und die künstlerische Weiterentwicklung auf der Stelle tritt.

\section{Intermezzo: Das Beinahe-Ende einer Karriere}

Die 1980er Jahre schließlich werden für Sylvie Vartan zu einer Zeit des Umbruchs: Zwar hat sie mit Chansons wie „L'amour c'est comme une cigarette“ (1981) oder „Toute une vie passe“ (1983) noch einige größere Erfolge und tritt bei großen Shows in Las Vegas auf 
(1982/83), doch gehen die Verkaufserfolge Mitte des Jahrzehnts deutlich zurück. Daran ändern auch französische Versionen englischsprachiger Hits wie „Danse ta vie“ („Flashdance“) oder „Déprime“ („Sweet dreams“) wenig. Nachdem 1986 der langjährige Plattenvertrag ausläuft, denkt Vartan gar darüber nach, sich aus dem Showbusiness zurückzuziehen. Hierfür spielen zum einen private Gründe eine Rolle (nachdem sie Anfang der 1980er Jahre den Produzenten Tony Scotti kennengelernt und ihn 1984 geheiratet hat, übersiedelt sie zusammen mit ihrer Familie nach Kalifornien), zum anderen aber auch das Fehlen einer neuen musikalischen Perspektive und der deutlich nachlassende Erfolg. Vartan selbst kommentiert dies so: „[...] au milieu des années quatre-vingt [...] je sentais que les choses ne tournaient plus. On ne me proposait rien d'enthousiasmant, j'avais l'impression de faire partie des meubles, et à ce titre d'enregistrer un album par an sans projet ni objectif." (Vartan 2007, 217) Sylvie Vartan ist mit diesem Phänomen nicht alleine, denn auch Größen des Chansons wie Charles Trenet oder Juliette Gréco tun sich in den späten 1970er und frühen 1980er Jahren schwer, an alte Erfolge beim Publikum anzuknüpfen bzw. bei Konzerten die Säle zu füllen. Die ,Années Top 50' in den 1980er Jahren, die mit einer neuen Form der Hitparade unzählige neue InterpretInnen auf den Musikmarkt drängen - aus denen Namen wie JeanJacques Goldman, Patrick Bruel, Étienne Daho, Marc Lavoine, Mylène Farmer oder Patricia Kaas herausragen -, machen es der ,alten Garde` schwer, sich im wahrsten Sinne des Wortes Gehör zu verschaffen. Parallel zu den Jahren des chanson yé-yé prägen in den 1980er Jahren nach der Liberalisierung der Radiofrequenzen ${ }^{10}$ die Medien die Chansonlandschaft, indem sie immer neuen SängerInnen zu Popularität in der Hitparade „Top 50“ verhelfen. Analog zu den 1960er Jahren lässt sich dabei beobachten, dass auch hier vielen InterpretInnen nur ein oder zwei Hits gelingen, aber selten dauerhafte Karrieren ihren Anfang nehmen. Für Sängerinnen wie Sylvie Vartan ist indes kein Platz in den „Top 50“, zumal weder ihr Musikstil kompatibel mit dem neuen Sound ist noch sie für sich in Anspruch nehmen kann, ein Vorbild für die Jugendlichen der 1980er Jahre zu sein: Zu groß ist mittlerweile der Altersunterschied, aufgrund dessen Vartan zur Generation der Eltern eben dieser Jugendlichen zählt.

Der Rückzug Sylvie Vartans dauert indes nur einige wenige Jahre, denn 1989 veröffentlicht sie ein neues Album mit dem Titel Confidanses und gibt 1990, inmitten der politischen Umwälzungen in Osteuropa, ein Konzert in ihrer Heimatstadt Sofia. Das Echo in den französischen Medien ist freilich deutlich geringer als in den 1960er Jahren.

\section{Phase II: Vartan und die nouvelle génération}

Mitte der 1990er Jahre versucht Sylvie Vartan schließlich eine künstlerische Neuausrichtung, indem sie mit Komponisten und Autoren einer jüngeren Generation zusammenarbeitet. Nach dem Album Vent d'Ouest von 1992 erscheint 1996 die Produktion Toutes les femmes ont un secret, die sich durch Texte von hoher Qualität auszeichnet. Diese stammen u.a. von Luc Plamandon („Je n'aime encore que toi“), Julie Daroy („Toutes les femmes ont un secret“), Jean-Louis Murat („Jour d'hiver") und Lyvia d'Alché („Des mots d'amour"). 
Die CD schafft das erste Mal seit langer Zeit den Status einer goldenen Schallplatte, doch ist unübersehbar, dass das Medieninteresse dem Vergleich mit den 1960er Jahren keineswegs standhält. Nach einem weiteren Album mit dem Titel Sensible aus dem Jahr 1998 startet Vartan zur Jahrtausendwende ein ambitioniertes Projekt mit dem Titel Tour de siècle: Neben ihren eigenen Chansons interpretiert sie Klassiker des Chansons u.a. von Léo Ferré, Édith Piaf, Jacques Brel und Barbara. Vartan selbst begründet dieses Projekt so: „J'interpréterai toutes ces chansons qui racontent mieux la France que les meilleurs livres d'histoire. J'ai toujours aimé chanter et faire miennes les chansons des autres, pour peu qu'elles trouvent un écho dans mon cœur." (Vartan 2004, 392) Die anschließende Tournee wird zum Erfolg, wenngleich nicht übersehen werden darf, dass das Publikum Vartans in der Mehrzahl mit ihr älter geworden ist und sich aus den Jugendlichen der Zeiten des chanson yé-yé zusammensetzt. Nachdem Vartan 2009 auf dem Album Toutes peines confondues bereits das Chanson "Je chante le blues"von Carla Bruni gecovert hatte, sorgt sie 2010 mit dem Album Soleil bleu für eine Überraschung: Das Gros der Chansons stammt von AutorInnen der jungen Generation, wie etwa Doriand und Keren Ann (,J'fais la moue“, „Je me détacherai“, „Le monde est moins dur que toi“), La Grande Sophie („Personne“), Julien Doré („Soleil bleu“) oder Benjamin Biolay („La vanité"). Sylvie Vartan zeigt sich damit von einer neuen Seite, jedoch offenbart das Album zugleich ein Dilemma: „Personne n'a voulu de son CD baptisé justement Personne ${ }^{11}$, de même que le suivant, Nashville en 2013! Sauf en 2007 où elle a eu la bonne idée de reprendre d'anciens tubes yé-yé dans un CD justement baptisé Nouvelle vague [...]" (Jelot-Blanc 2014, 155). In der Tat bleiben die Verkaufszahlen deutlich hinter den Erwartungen zurück, was sich damit erklären lässt, dass die Fans der "nouvelle génération“ die Chansons lieber von AutorInnen und KomponistInnen wie Keren Ann oder La Grande Sophie selbst hören möchten, die Fans von Sylvie Vartan aber ihr klassisches Repertoire mit den Hits aus den 1960er und 1970er Jahren bevorzugen. Dieser Spagat ist schwerlich zu bewältigen, wie der relative Misserfolg des durchaus gelungenen Albums zeigt. Greift die Interpretin hingegen Chansons aus ihrer Glanzzeit auf, wie auf dem bereits erwähnten Album Nouvelle vague von 2009, auf dem sie u.a. „Il est cinq heures, Paris s'éveille“ von Jacques Dutronc, „Attends ou va-t-en“ von Serge Gainsbourg, „Suzanne“ von Leonard Cohen oder „I'm a believer" von Neil Diamond interpretiert, folgt ihr das Publikum in die Konzertsäle und kauft die CD. Vor diesem Hintergrund kann es nicht verwundern, dass Vartan bereits mehrere Alben herausgebracht hat, auf denen sie die Klassiker ihres Repertoires in neuen Arrangements interpretiert bzw. in regelmäßigen Abständen Live-Alben veröffentlicht, die viele dieser Klassiker enthalten. Das Ende November 2015 erschienene, bislang letzte Album Une vie en musique, das in die drei Kategorien "Sofia“, „Paris“ und "Los Angeles“ unterteilt ist, passt denn auch genau in diese Schiene: Seine insgesamt 16 grundlegenden Chansons aus dem Repertoire der Sängerin bilden ein repräsentatives Resümee aus fünf Jahrzehnten Arbeit mit dem und im Chanson. 


\section{Revival des chanson yé-yé- und auch Sylvie Vartans?}

Zum Abschluss dieser Betrachtungen bietet es sich an, noch einen Blick auf ein Phänomen zu werfen, das als eine Art, Wiederbelebungsversuch' des chanson yé-yé verstanden werden kann: Die Rede ist von den Tourneen Âge tendre et têtes de bois, die ab 2005 von dem Manager Michel Algay ins Leben gerufen werden und sich über mehrere Jahre in Frankreich großer Beliebtheit erfreuen. Das Konzept besteht - in Anlehnung an den Titel eines Chansons von Gilbert Bécaud aus den 1960er Jahren - darin, eine Reihe von InterpretInnen mit ihren großen Hits aus vor allem eben diesem Jahrzehnt für eine Serie von Musikgalas gemeinsam auf die Bühne zu stellen und damit von der Nostalgiewelle zu profitieren, die sich in den 2000er Jahren in Europa in unterschiedlichen Bereichen breit macht. ${ }^{12}$ Es verwundert indes nur wenig, dass vor allem Sängerinnen und Sänger, deren Glanzzeiten schon länger vorbei sind, zu den Teilnehmern an diesem Projekt zählen. So finden sich in den ersten Jahrgängen Namen wie Frank Alamo, Sheila, Michèle Torr, Gilles Dreu, Pascal Danel, Richard Anthony oder auch Rika Zaraï, die allesamt zu Zeiten des chanson yé-yé und in den Jahren danach beliebte Stars waren. ${ }^{13}$ Das Projekt ist so erfolgreich, dass nach der ersten Tournee im Jahr 2006 bis in die Saison 2013-14 eine alljährliche Neuauflage folgt, was damit einhergeht, dass auch die französischen Medien die Zeit des chanson yé-yé wiederentdecken und ihm zahlreiche Sendungen widmen, wie die beiden folgenden Beispiele zeigen: „Le 15 janvier 2007, la jeune animatrice Frédérique Courtadon est chargée de présenter sur France 3 un autre hommage baptisé Le Temps des yé-yé. [...] Enfin à l'été 2010, Arte diffuse Yé yé révolution 1962-1966, une émission documentaire de Michel Royer et Didier Varrod [...]“ (JelotBlanc 2014, 112). Die Tatsache, dass man sich in jenen Jahren auf den 50. Jahrestag der Entstehung des Phänomens yé-yé zubewegt, erweist sich als zusätzliches verkaufsförderndes Element, das von den Medien bereitwillig aufgegriffen wird. Dies alles kann freilich nicht darüber hinwegtäuschen, dass sich der Großteil des Publikums aus Altersschichten zusammensetzt, die in ihrer Jugend Fans dieser Musikrichtung und ihrer Stars waren und der Nostalgie jener Zeit (und vielleicht ihrer eigenen Jugend) nachhängen. Ein dauerhaftes Interesse beim jüngeren Publikum lässt sich - vor allem auch in Bezug auf die SängerInnen - nicht wirklich erkennen, wenngleich einige Altstars noch einmal in den Genuss von (kurzfristigem) Glanz kommen: „[C’est] une cure de jouvence, une sacrée revanche aussi pour toutes ces anciennes vedettes déchues, naguère traitées de has been! Partout où ils se produisent aux quatre coins de France, les attend toujours la même frénésie dont ils s'étonnent encore et toujours [...]" (Jelot-Blanc 2014, 113).

Interessant ist allerdings, dass die noch lebenden großen Stars des chanson yé-yébei diesen Galas durch Abwesenheit glänzen: Weder Johnny Hallyday noch Françoise Hardy wirken an dem Revival mit, genauso wenig wie Sylvie Vartan, die in jenen Jahren die oben beschriebene Annäherung an die jüngere Generation des Chansons versucht und sich auf kleinere Konzertsäle verlegt, wie das Pariser Olympia oder die Salle Pleyel, in der sie $2011 \mathrm{ihr}$ 50-jähriges Bühnenjubiläum feiert. Die Begleitung durch das bulgarische Philharmonische Orchester sorgt indes dafür, dass sich selbst die ,alten 'Chansons aus der Zeit des yé-yé in 
einem musikalisch völlig anderen Gewand präsentieren. Hinzu kommt eine deutlich sparsamere mise en scène, die auch nichts mehr mit den großen Shows der 1970er Jahre zu tun hat, sondern eher an den klassischen tour de chant großer Interpretinnen des Chansons wie Édith Piaf oder Juliette Gréco erinnert. „La Sylvie aux couettes est définitivement devenue la grande Vartan“" (Saka/ Plougastel 1999, 425) ist denn auch in einem der Nachschlagewerke zum französischen Chanson nachzulesen; der Satz mag sicherlich insofern seine Berechtigung haben, als es Sylvie Vartan gelungen ist, ihrem Repertoire eine eigenständige Richtung zu geben, anstatt sich ausschließlich an immer neuen musikalischen Tendenzen zu orientieren.

Damit schließt sich der Bogen zu den Ausgangsfragen: Kann Sylvie Vartan der Wandel von einem Zeitgeistidol des chanson yé-yé zu einer grande dame des Chansons attestiert werden? Das Beispiel Vartan zeigt auf jeden Fall, in welchem Maß die Medienpräsenz bestimmter Stars einen wesentlichen Einfluss auf die musikalische Karriere nimmt, wenngleich auch deutlich wird, dass gerade Musikströmungen, die einer so starken Modewelle unterworfen sind wie das chanson yé-yé, einem gewissen Verfallsdatum unterliegen. Eine musikalische Weiterentwicklung ist für die Vertreter solcher Modeströmungen also unabdingbar, funktioniert aber nicht immer. Im Falle Sylvie Vartans gelingt dies mit den großen Bühnenshows in den 1970er Jahren gerade deshalb recht gut, weil auch diese wieder dem Zeitgeist entsprechen; spätestens ab der 1980er Jahren jedoch hat Vartan zunehmend Mühe, den musikalischen Geschmack der breiten Masse zu treffen. Zwar kann sie nach wie vor auf ein gewisses treues Publikum bauen, doch wird der Stamm der Fans mit den Jahren deutlich kleiner. Es wäre insofern interessant zu vergleichen, wie sich diese Phänomene beispielsweise bei Johnny Hallyday, einem der anderen großen Namen des chanson yé-yé und zusammen mit Sylvie Vartan Medienliebling der 1960er Jahre, auswirken.

\section{Endnoten}

1 Der Begriff, chanson yé-yé geht zurück auf den französischen Soziologen Edgar Morin, der ihn in einem Zeitungsartikel von Le Monde im Jahr 1963 zum ersten Mal benutzt. Er leitet sich von den „yeah-yeah“-Rufen ab, die zahlreiche Songs angloamerikanischer Herkunft aus jenen Jahren prägen. Morin prägt darüber hinaus den Begriff der ,décagénaires‘, um das englische Wort ,teenager` zu übersetzen (Calvet 2013, 140).

2 Der Begriff, Trente Glorieuses' bezeichnet die Jahre des wirtschaftlichen Aufschwungs in Frankreich in der Zeit vom Ende des Zweiten Weltkrieges bis zur ersten Ölkrise Mitte der 1970er Jahre.

3 So schreibt Aznavour u.a. für Sylvie Vartan „La plus belle pour aller danser“, während Serge Gainsbourg der jungen France Gall mehrere Chansons auf den Leib schneidert (z.B. „Les sucettes à l'anis").

4 Für eine ausführlichere Analyse der Mediatisierung der Interpretinnen, die den Rahmen dieses Beitrags sprengen würde, sei verwiesen auf Kapitel V.5 „Mediatisierung der Interpretinnen und damit verbundene Muster von Weiblichkeit“ in Bonnermeier 2002, 215-231. 
5 Die Begriffe ,real person', ,performance persona' und ,character' (im Sinne des Protagonisten oder der Protagonistin eines Chansontextes) werden von dem Kulturwissenschaftler Philip Auslander, der sich auf den Bereich der Performanz spezialisiert hat, in mehreren Aufsätzen geprägt und definiert. Cf. Auslander 2004, 6.

6 http://atilf.atilf.fr/dendien/scripts/tlfiv5/advanced.exe?8;s=1253395380 (Zugriff 06.10.2016).

7 http://atilf.atilf.fr/dendien/scripts/tlfiv5/advanced.exe?8;s=1253395380 (Zugriff 06.10.2016).

8 Ein zeitgenössischer Eindruck von Sylvie Vartan als Werbeträgerin für die Haute couture von Dior findet sich in Vartan 2007, 54-55.

9 Johnny Hallyday wird diese ,Ehre' bereits im Jahre 1964 zuteil; seine Fotografie wird zudem unter der Überschrift „L'idole des jeunes“ auf der Titelseite der Zeitschrift Nous deux abgedruckt. Andere Stars des chanson yé-yé wie Frank Alamo oder Rika Zaraï folgen (Dillaz 2005, 123-124).

10 Die ersten privaten Radiosender werden Ende 1984 unter der Präsidentschaft von François Mitterrand autorisiert, was in den Folgejahren zu einem regelrechten Boom von neuen Sendern führt.

11 Was nicht korrekt ist: Das Album heißt Soleil bleu.

12 Man denke in diesem Zusammenhang z.B. an den Erfolg diverser Automodelle im Retro-Stil wie Fiat 500, Mini oder VW Beetle oder aber auch an die Vintage-Tendenzen im Bereich der Wohnungseinrichtung.

13 Allerdings werden auch InterpretInnen in das Projekt integriert, die zur Zeit des chanson yé-yé ihre Karriere noch gar nicht begonnen haben, wie beispielsweise Patrick Juvet, Marie Myriam, Herbert Léonard oder Demis Roussos, oder bereits zuvor im Geschäft waren wie Charles Dumont oder Annie Cordy.

\section{Bibliographie}

Auslander, Philip: „Performance Analysis and Popular Music: A Manifesto“. In: Contemporary Theatre Review 14,1 (2004), 1-13, homes/mc.gatech.edu/-auslander/publications/performance analysis.pdf (Zugriff 29.09.2016).

Bonnermeier, Andreas: Frauenstimmen im französischen Chanson und in der italienischen Canzone. Ein Genre und seine Interpretinnen. Hamburg: Dr. Kovac, 2002.

Bügler, Elisabeth: „Stars et antistars. Comment le chansonnier actuel comprend-il son métier? Conceptions et mise en pratique dans la métachanson“. In: Rieger, Dietmar (Hg.): La chanson française et son histoire. Tübingen: Gunter Narr, 1988, 363-394.

Brunel, Pierre (Hg.): Dictionnaire des mythes d'aujourd'hui. Monaco: Éditions du Rocher, 1999.

Calvet, Louis-Jean: 100 ans de chanson française (1907-2007). Paris: L'Archipel, 2006.

Calvet, Louis-Jean: Chansons. La bande-son de notre histoire. Paris: L'Archipel, 2013.

Dillaz, Serge: Vivre et chanter en France. Tome 1 1945-1980. Paris: Fayard/Chorus, 2005.

Jelot-Blanc, Jean-Jacques: Yé-yé-Idoles ou Has-been? o.O.: Éditions Vaillant, 2014.

Hölz, Karl: „La chanson moderne et les mass-média. Une étude thématique et sociocritique“. In:

Rieger, Dietmar (Hg.): La chanson française et son histoire. Tübingen: Gunter Narr, 1988, 347-362. 
Lesouëf, Bruno (Hg.): 1945-1975 Les Trente Glorieuses. La France heureuse. Paris: Sophia Publications, 2013.

Oger, Armelle: C'était comme ça en France. 1945-1975 Les Trente Glorieuses. Paris: Éditions Gründ, 2013.

O.A.: „Idole“. In: http://atilf.atilf.fr/dendien/scripts/tlfiv5/advanced.exe?8;s=1253395380 (Zugriff 06.10.2016).

Saka, Pierre / Plougastel, Yann: La chanson française et francophone. Paris: Larousse, 1999.

Saka, Pierre: La grande anthologie de la chanson française. Paris: Libraire Générale Française, 2001.

Vartan, Sylvie: Entre l'ombre et la lumière. Paris: XO Éditions, 2004.

Vartan, Sylvie: Dans la lumière. Paris: XO Éditions, 2007.

\section{Diskographie}

Vartan, Sylvie: Vent d'Ouest, Phonogram France/ Philips 512994-2, 1992 (CD).

Vartan, Sylvie: L'essentiel (Compilation), RCA/BMG France 74321262312,1995 (CD).

Vartan, Sylvie: Toutes les femmes ont un secret, Mercury/ Philips 528895 2, 1996 (CD).

Vartan, Sylvie: Sensible, Mercury/ Philips 538 117-2, 1998 (CD).

Vartan, Sylvie: Sylvie, Mercury/ Universal 981846 1, 2004 (CD).

Vartan, Sylvie: Nouvelle vague, Mercury/ Universal 530 042-5, 2007 (CD).

Vartan, Sylvie: Toutes peines confondues, RCA/ Sony Music 8869751112, 2009 (CD).

Vartan, Sylvie: Soleil bleu, RCA/ Sony Music 88697778312, 2010 (CD).

Vartan, Sylvie: Live à la salle Pleyel, RCA/ Sony Music 869197492, 2011 (CD).

Vartan, Sylvie: Une vie en musique, Sony Music88875167042, 2015 (CD). 